\title{
Educación física: ENTRE las nuevas demandas del ejercicio profesional y la formación de grado
}

\author{
Ivana Verónica Rivero \\ Universidad Nacional de Río Cuarto, Rio Cuarto, CÓRDOBA, Argentina. \\ Silvia Beatríz Libaak \\ Universidad Nacional de Río Cuarto, Rio Cuarto, CÓRDOBA, Argentina.
}

\section{Resumen}

El presente escrito expone los resultados finales del proyecto de investigación ${ }^{1}$ desarrollado por un equipo de investigación del Departamento de Educación Física de la Universidad Nacional de Río Cuarto durante los años 2012 y 2013. El objetivo de esta investigación cualitativa, interpretativa fue conocer la relación entre las actuales demandas laborales del ejercicio profesional del docente de educación física y su formación de grado. Se entrevistaron en profundidad a profesores insertos laboralmente en distintos contextos laborales y dedicados a la enseñanza de diferentes prácticas corporales. Del análisis de estas entrevistas surgieron cuatro resultados finales con sus respectivas conclusiones, las cuales se detallan en el presente artículo.

Palabras Clave: Educación física; formación docente; ejercicio profesional; demandas

\section{Contextualización de la investigación}

La educación física argentina de hoy se encuentra atravesada por nuevas demandas de la sociedad que tensionan sentidos y prácticas instituidos en la cultura corporal. Por un lado, el consumo pasivo del deporte (desde la observación y no desde la acción), el bombardeo mediático de patrones corporales inalcanzables, los desbordes populares en eventos deportivos, entre otras situaciones obligan a replantear las experiencias de prácticas corporales cotidianas y a buscar nuevas oportunidades. Por otro lado, la creciente demanda de propuestas corporales que contrarresten los efectos de una vida sedentaria en las distintas franjas etarias, la necesidad de acercar las propuestas de actividad física a las posibilidades de cada persona, y la pretensión de hacer masiva la participación de la

Proyecto de Fomento a la Investigación aprobado por SeCyT UNRC, Res. Rec. N852/11 y financiado por Res. Rec. $\mathrm{N}^{\circ} 328 / 12$. Código E337. Integrantes del equipo de investigación: Ivana Rivero (Directora), Silvia Libaak, Verónica Picco, Viviana Gilleta, Claudio Aruza, Sergio Centurión, Manuel Barcelona, Daniel Faro, Roberto Fernández, Luciana Leiggener, Matías Zarauz. Becarios: Manuel Limbrici, Jennifer Seisdedos, Natalí Esteves, Gastón Vicentini, Federico Serra. 
sociedad en propuestas de actividad física, han ampliado considerablemente los ámbito y posibilidades de intervención del docente de Educación Física.

Es así que en la actualidad las propuestas profesionales de la educación física incluyen gran variedad de prácticas corporales. Algunas están amparadas en la tradición como los deportes, la gimnasia, la natación, los juegos y actividades de vida en la naturaleza. Otras prácticas, se apoyan en propuestas innovadores o alternativas amparadas en su gran mayoría por las nuevas tendencias juveniles.

Atendiendo a este proceso de acomodo de las prácticas a las preferencias personales, numerosas empresas privadas han encontrado una oportunidad para comercializar credenciales habilitantes que aficionados y algunos graduados suelen tomar para acceder a puestos laborales no formales en clubes, centros recreativos, escuelas deportivas, gimnasios, centros de rehabilitación y estética, colonias de vacaciones, etc.

Así, propuestas de formación breves a altos costos con escasos requisitos para el ingreso y organizadas por empresas privadas, han facilitado que las nuevas demandas de actividad física sean igualmente atendidas por aficionados, ex deportistas, personal trainers, entrenadores, técnicos, instructores, que por profesionales docentes de educación física. Si a esta situación se suma que en la escuela (institución educativa formal), espacio de intervención regulada y protegida para profesionales docentes, las vacantes de trabajo están saturadas, se concluye en que los recientes graduados de educación física encuentran un escenario laboral complejo y difuso, que los impulsa a buscar un lugar en el ámbito educativo no formal.

Tal como sostiene Centurión (1997), el campo laboral de los profesores de Educación Física se ha ampliado considerablemente en el ámbito no formal y es dificultoso mantener una alta valoración de la tarea y eficiencia en este ámbito; perdiéndose de este modo, terreno laboral frente a personas con diferente capacitación y experiencia. Esto es porque las nuevas tendencias y reactualizaciones de prácticas corporales, influenciadas por la moda, requieren de nuevas respuestas que obligan a revisar las matrices de formación de la Educación Física (Cachorro, 2013). Proceso lento que siempre llega con retraso a una demanda siempre cambiante.

Las distintas instancias de encuentro de profesores de educación física ponen a luz la necesidad de discutir las relaciones que se entretejen entre las nuevas demandas y la formación necesaria para un ejercicio profesional de calidad.

De aquí el objetivo de esta investigación: conocer y comprender las relaciones entre la formación docente y el ejercicio profesional del docente de Educación Física.

\section{Decisiones teórico metodológicas}

Un abanico de conceptos sensibilizaron la búsqueda de indicios que permitieron conocer y comprender las relaciones que se trazan entre formación de grado y ejercicio profesional del docente Educación Física. Entre ellos, el concepto bourdieano de 'campo', como el lugar de luchas donde se construyen sentidos comunes y los agentes empeñan el capital que adquirieron en luchas anteriores, sus estrategias e intereses para transformar o conservar las relaciones de fuerza (Bourdieu, 1996). 
En el estudio del movimiento corporal con sentido y significado cultural (Bracht, 1996; Giraldes, 2001; Lopes de Pavia \& Ron, 2009), la Educación Física se constituye epistemológicamente en el atravesamiento de diferentes campos de conocimiento con pretensiones científicas que, de algún modo, disputan su hegemonía. La mirada particular que la fisiología, la psicología, la pedagogía, la sociología, entre otros, han hecho y hacen del movimiento corporal, entran en relación asignando al campo una constitución fragmentada solo superable si quienes eligen formarse en esta área de conocimiento reconocen que, como afirma Bracht (1999), su legitimidad proviene más del reconocimiento que la sociedad hace de la actuación profesional, que de su fundición con la ciencia.

Autores como Bracht $(1996,1999)$, Fensterseifer (2000), Vaz (2009), y Remedi (1999) defienden la idea de la Educación Física como práctica que, por ser pedagógica, involucra una dimensión ética, ya que siempre hay un alguien que hace una valoración sobre lo enseñable, lo enseñado, lo que se desea enseñar.

Esta postura lleva a preguntar, si la docencia es considerada una profesión. En este sentido, Tenti Fanfani sostiene que "Desde un punto de vista sociológico, se considera que un trabajo o una actividad es 'profesional' si, entre otras cosas, el que lo realiza tiene una calificación especializada, la que se expresa en los títulos que dispensa el sistema educativo formal" (2005, pág. 61).

Barrow y Brown (1992) en coincidencia con otros autores, afirman que al menos siete criterios permiten distinguir una profesión de un oficio, entre ellas, un período prolongado de formación y destrezas especializadas contenidas en el corpus de conocimiento de una disciplina académica (libros, revistas, publicaciones en general) que los miembros llevan a cabo.

Recuperando palabras de Gómez Campo y Tenti Fanfani: "La formación profesional introduce inevitablemente una suerte de transformación (de formación, adaptación o desarrollo específico) de sus cualidades perceptivas, ya que los conocimientos y/o experiencias adquiridas configuran el conjunto de las categorías, valores y criterios a través de los cuales percibe, comprende, interpreta e interviene en la realidad" (1989, pág. 14). Davini sostiene que los docentes pasan por "ambientes de modelación de las prácticas y del pensamiento" (Davini, 2005, pág. 79).

Los interrogantes que guiaron esta investigación fueron: ¿cuáles son las características de los nuevos contextos laborales y aquellos reconocidos como propios del campo?, ¿qué saberes son necesarios para ejercer la profesión en los diferentes contextos laborales?, ¿qué particularidades reviste el ejercicio profesional del docente de Educación Física en la enseñanza de las prácticas corporales en distintos contextos laborales?; en esta dirección, la formación docente de grado de la UNRC ¿responde a las demandas laborales del contexto?, ¿brinda a los futuros docentes las herramientas necesarias para adecuar sus saberes a los requerimientos del ejercicio profesional?

Esta investigación de tipo cualitativa, se inscribe en el 'paradigma interpretativo' (Vasilachis, 1992a). Los antecedentes teóricos existentes sensibilizaron, orientaron y dieron a conocer el estado de conocimiento respecto a las relaciones entre la formación de grado y el ejercicio profesional de los docentes de Educación Física, pero de ningún modo constituyeron categorías a seguir en la investigación (Strauss y Corbin, 2002) El cuerpo de la investigación se construyó a partir de interrogantes que, por abducción, sur- 
gieron a partir de los datos y orientaron la discusión sobre los aspectos teóricos más constantes (Vasilachis, 1992a).

Para acceder al sentido de las prácticas se realizaron entrevistas en profundidad (Tylor y Bogdan, 1987; Vasilachis, 1992), a docentes de Educación Física insertos laboralmente seleccionados por muestreo teórico.

Esta investigación se amparó en algunos supuestos. El primero de ellos afirma que existe un desfasaje entre los saberes construidos durante la formación de grado del docente de Educación Física y los saberes requeridos para responder a las demandas que la sociedad requiere de la profesión. El segundo supuesto es que los docentes cubren ese desfasaje buscando formación alternativa que no garantiza calidad en la enseñanza de prácticas corporales, de modo que, el ejercicio profesional del docente de Educación Física aparece hoy ligado a aspectos vinculados a la vocación y el oficio más que a la profesionalización. El tercer supuesto es que durante el cursado de materias vinculadas a las 'prácticas corporales' (Gayol, 2013; Gallo Cadavid, 2009; Crisorio, 2007) o 'configuraciones de movimiento' (Ron, 2001), como juego, deporte, gimnasia, vida en la naturaleza, expresión corporal, los futuros docentes construyen saberes que se redefinen en el ejercicio de la profesión, de acuerdo al contexto de intervención.

Finalmente, se partió del supuesto de que la posibilidad de desplegar el abanico de oportunidades laborales del docente de Educación Física, reconociendo los saberes requeridos para un desempeño de calidad profesional, favorece la sensibilización de los docentes universitarios para reflexionar y actuar en relación a la propuesta educativa vigente.

\section{Sobre el trabajo de campo}

Para discutir sobre las tensiones que se generan entre las demandas actuales y la formación de los docentes de Educación Física para un ejercicio profesional de calidad, fue necesario introducirse en el contexto de intervención profesional e involucrarse en la trama de relaciones que le otorga sentido a la problemática planteada. Para ello, a través de entrevistas en profundidad, se escucharon voces de distintos profesionales de educación física insertos en diferentes contextos laborales y vinculados a la práctica y enseñanza de distintas prácticas corporales.

En esta investigación, tuvo relevancia la perspectiva de los actores involucrados directamente. Por una cuestión de ética en la investigación, previo a la realización de las entrevistas se ofreció a los docentes garantía de anonimato, pues en ellas se encuentran declaraciones personales en las que los sujetos comparten sus creencias, actitudes y valores. Además se intentó interpretar el punto de vista del otro acerca de la realidad lo más cercano posible a como ellos la viven y la sienten.

Así, la voz de los docentes de Educación Física permitió acceder al modo en que resuelven las tensiones que se generan entre la formación recibida y los requisitos para la inserción laboral a diferentes instituciones, y comprender su perspectiva. Al entrar en su propio mundo, en su situación, en su contexto, en su historia (Guber, 2001; Vasilachis, 2003), se abrió camino hacia la interpelación de afirmaciones hechas por estudiosos seleccionados respecto de sus aportes teóricos. 
Las entrevistas se realizaron con diálogos distendidos y relajados, mediante los cuales los entrevistadores procuraron acceder al punto de vista de los entrevistados. Las preguntas giraron en torno a cinco ejes temáticos seleccionados a partir de los antecedentes teóricos de la investigación: relaciones entre la práctica y la Educación Física, formación profesional, la práctica corporal en cuestión, los practicantes y la didáctica de la práctica.

Para las entrevistas en profundidad (las cuales fueron desgravadas en forma de texto), se utilizó el reconocimiento de los 'puntos de anclaje de la subjetividad' guiados por la 'teoría de la enunciación', centrando la atención en unidades subjetivas, en huellas lingüísticas del enunciador, de sus modos de existencia, lo que Benveniste llama la subjetividad en el lenguaje y Kerbrat- Orecchioni reconoce como lingüística de la enunciación en sentido restrictivo, ya sean elementos del nivel sintáctico, semántico o pragmático (Kerbrat Orecchioni, 1997). En algunos fragmentos se analizaron los sentidos explícitos, literales de los conceptos seleccionados por el enunciador para hacer explícita una idea o el modo en que se organizan esos conceptos jerarquizando la información. En otros fragmentos se accedió al funcionamiento de los conceptos en el texto en particular, es decir, al sentido latente, sugerido de los diferentes conceptos en el texto por medio de las connotaciones simbólicas, especialmente los adjetivos.

Así, siguiendo diferentes caminos en función de las posibilidades encontradas en el análisis de cada documento, se buscaron y analizaron marcas textuales y ausencias, huellas de producción para encontrar ideas recurrentes que favorecieran el acceso a las ideas del enunciador que, al compartirlas con sus pares, representan a la comunidad discursiva.

Luego, por el 'método de constante comparación', se fueron quebrando los datos y reorganizándolos en ideas recurrentes que permitieron, dentro de ellas y entre ellas, la comparación y contrastación. Señalando diferencias y similitudes se pudieron conformar grandes ejes de discusión e identificar en ellos diferentes posturas (Glaser \& Strauss, 1967).

Las categorías y propiedades que surgieron del análisis de los datos fueron plasmadas en planillas que permitieron una mejor visión de los puntos sensibles para poner en discusión con textos intencionalmente seleccionados para el análisis de los resultados.

\section{Resultados}

A partir de este análisis de las entrevistas se identificaron cuatro resultados finales:

1. Se pueden identificar nuevas prácticas laborales que, sin tener escenarios prefigurados, se constituyen en nuevas oportunidades para el ejercicio profesional del docente de Educación Física.

El campo de la Educación Física es dinámico, en constante trasformación; así en las voces de los entrevistados se identificaron nuevas demandas, problemas y posibilidades. Tal como sostiene Ron $(2005,2013)$ la educación física además de ser una práctica 
pedagógica es una práctica social y como tal, es la sociedad a través de sus actores sociales quien le asigna sentido y significado.

Según las voces de los entrevistados los saberes incorporados en la formación de grado no siempre posibilitan la aplicación directa en los nuevos escenarios laborales. En la búsqueda de oportunidades algunos docentes logran estabilidad laboral en escenarios conocidos como gimnasios, clubes, centros de rehabilitación; y otros, trazan recorridos no amparados en la formación que van desde la coordinación de murgas, enseñanza de habilidades corporales artísticas hasta actividades corporales en escenarios naturales como ascensos a cerros, cicloturismo, traiking entre otras prácticas.

Las voces de los entrevistados comparten la idea de que en el ejercicio de la profesión los saberes construidos se ponen a prueba constantemente, se reactualizan, y demandan formación permanente.

Este resultado permite formular la siguiente conclusión: la profesionalidad es un proceso continuo que no se agota en la formación inicial. En esta dirección, la formación docente no sólo debe formar para enseñar, sino además para investigar y diseñar modos particulares de intervenir y gestionar en ámbitos y contextos no escolares.

2. Ser experto en una práctica corporal requiere conocer la gente que se dedica a ella, ya sea practicándola como enseñándola.

Los docentes entrevistados reconocieron que para hacer popular una práctica es necesario difundirla y eso requiere de otras personas que se dediquen a ella. Esas personas constituyen un círculo de especialistas sobre el tema que se presenta como un círculo de interlocución válida, pues con ellos consiguen entenderse, en palabras de los entrevistados "hablan el mismo idioma", "con ellos no hace falta explicar tanto porque saben de lo que estamos hablando".

Este resultado permitió llegar a la conclusión de que la profesionalidad del docente de Educación Física se asienta en compartir espacios, problemáticas, dudas, experiencias con colegas. En este sentido, se vuelve una necesidad generar espacios de estudio, diálogo y discusión entre profesionales de la Educación Física para estimular el proceso de formación permanente. Así, se refuerza la idea de formación como proceso inacabado, abierto, que deja en evidencia que la identidad profesional es una construcción continua (Giles, Molina Neto, \& Kreusburg Molina, 2005).

En esta construcción de identidad profesional cobra gran importancia el encuentro con el otro, con la comunidad docente, especialmente con el colega profesional, pues permite socializar las experiencias pedagógicas, reformular conceptos, posicionamientos, estrategias entre otros. Según refiere Molina Neto, la generación de distintos espacios de interlocución en la educación física permite hacer formación a partir de las experiencias de los profesores (Ruiz, 2004).

De este modo, el encuentro, dialogo y debate entre los profesionales dedicados a una misma práctica corporal puede ayudar a una reconstrucción de la formación docente, permitiendo la trasmisión de procedimientos didácticos, de recetas de cómo solucionar problemas comunes, de identificar obstáculos y desafíos, de elaborar de manera conjunta estrategias de acciones educativas en el campo disciplinar. Así, a partir de la divulgación 
de las experiencias docentes, muchas de las experiencias positivas pueden ser aprovechadas y transferidas a distintos escenarios educativos.

En esta relación grupal, la capacidad de escucha es una competencia necesaria para el profesional de educación física, en los diferentes ambientes; ya sea como estrategia de enseñanza o como modo de encaminar sus investigaciones (Giles, Molina Neto, \& Kreusburg Molina, 2005). A partir de este resultado, desde esta investigación se propone la 'capacidad de compartir', pues no basta con escuchar sino con tener 'algo' (un conocimiento, una reflexión, sensación, una receta) para compartir y compartirlo, es decir, decirlo, mostrarlo, enseñarlo a los demás.

3. Se aprende a enseñar prácticas corporales siendo alumno de otro profesor, coordinador o aficionado, y mejor es el enseñante mientras más tiempo le dedique.

Los profesores entrevistados coinciden en afirmar que saber jugar, practicar deporte, nadar, hacer gimnasia, bailar, se aprende haciendo. Es así que se reivindica el valor de estar, de probar, de comprometerse, involucrarse, se reivindica la experiencia.

Este resultado permitió formular la siguiente conclusión: ser un experto en una práctica corporal requiere primero tener la experiencia corporal de esa práctica, y luego en la reflexión y análisis para que impacte en la didáctica de la práctica. En esta dirección, los espacios de formación profesional deberían articular experiencia y reflexión, teoría y práctica, saber proposicional y saber práctico.

Como afirman Di Gregori \& Duran (2009), la vinculación entre conocimiento y acción es insoslayable: el hombre sólo puede asignar sentido a las cosas en tanto pueda actuar con ellas. Sólo será experiencia para quien se deja atravesar por ella, para quien se entrega a ella.

Una experiencia “...significa una completa interpenetración del yo y el mundo de los objetos y acontecimientos” (Dewey, 2000, pág. 21), donde no sólo el actor carga de sentido el objeto sino que se deja modificar por él. La persona que practica un deporte, por ejemplo, no sólo consigue teñir el juego con su forma de moverse, con las decisiones que toma, sino que en ese preciso instante, él mismo está cambiando, está aprendiendo y desaprendiendo saberes. Esto es porque, "'Aprender por la experiencia' es establecer una conexión hacia atrás y hacia adelante entre lo que nosotros hacemos a las cosas y lo que gozamos o sufrimos de las cosas, como consecuencia” (Dewey, 1997, pág. 124).

Se aprende a jugar jugando, a practicar deporte jugándolo, a bailar bailando pues las prácticas corporales son saberes prácticos.

Para que la formación universitaria de profesores de Educación Física tenga éxito, resulta entonces necesario considerar el cúmulo de experiencias previas individuales y que asisten a modo de antecedentes en el sostenimiento de una creencia sobre por qué o para qué estudiar cada práctica corporal. Este proceso se sitúa en la historia personal de cada uno y al tener herramientas experienciales distintas, puede complicar la posibilidad de construir cimientos simbólicos comunes. Aunque puede ser entendido una dificultad, es precisamente este desafío lo que le permite a la disciplina repensarse continuamente. 
4. Dedicarse a la enseñanza de una práctica corporal deja poco tiempo del día para leer y esto dificulta el reconocimiento y puesta a prueba de bibliografía especializada.

Este resultado permitió formular la siguiente conclusión: la didáctica de las prácticas corporales es el tema de vacancia que demanda producciones académicas en el campo de la Educación Física, reflexiones y análisis de la experiencia práctica concreta.

La escisión entre los profesionales que se dedican a la producción de conocimientos sobre una práctica corporal, y quienes se dedican a la enseñanza de esa práctica podría comenzar a cicatrizarse si los primeros pensaran, estudiaran y produjeran junto a los segundos a partir de las 'recetas' que se utilizan, lo hicieran para ayudar a mejorar sus prácticas; y además buscaran diferentes estrategias de divulgación.

La didáctica de las prácticas corporales es el tema de vacancia que demanda producciones académicas en el campo de la Educación Física. Esto se comprueba al ver como se duplica la participación de los docentes del campo en propuestas de formación permanente coordinadas por docentes que 'le ponen el cuerpo' al discurso que sostienen.

La marcada tendencia a no leer de los docentes expertos en las prácticas corporales, exige un doble esfuerzo para el profesional de la Educación Física que se dedica a investigar esa práctica: tener dominio de las prácticas académicas que permitan posicionar la práctica corporal como saber digno de ser pensado y dicho en y por la academia, y conservar la frescura de la oralidad y de las impresiones de la experiencia para ser interlocutor válido para la comunidad de expertos de la práctica. Esto exige tener la capacidad para hablar de esa práctica y entenderse tanto sea, con investigadores de otros campos (sosteniendo un discurso que nos represente y favorezca la autonomía del campo) como con colegas dedicados a la enseñanza, practicantes, apasionados, aficionados, padres y la sociedad en general.

\section{A modo de cierre}

Esta investigación se respaldó en un constante trabajo en equipo, a través del cual se puso a discusión las distintas instancias de esta práctica investigativa. Desde la identificación del problema, con su respectiva justificación y relevancia, la elaboración del contexto conceptual, el desarrollo del trabajo de campo hasta el análisis y discusión de los resultados, fue producto del aporte de los diferentes integrantes. Esta triangulación de agentes permitió garantizar cierto control de calidad en las diferentes interpretaciones que permitieron dar credibilidad a los resultados obtenidos.

Estos resultados han permitido por un lado, ratificar algunos de los supuestos de esta investigación: el primer supuesto sostenía que existe un desfasaje entre los saberes construidos durante la formación de grado del docente de Educación Física y los saberes requeridos para responder las demandas contemporáneas de la sociedad. El segundo supuesto refiere a que los docentes cubren ese desfasaje buscando formación alternativa vinculada a la vocación y al oficio más que a la profesionalización. Y el tercer supuesto sostiene que durante el cursado de la carrera, los futuros docentes construyen saberes en las materias vinculadas a las 'configuraciones de movimiento' (Ron, 2005), o a las 'prácti- 
cas corporales' (Gayol, 2009; Crisorio, 2007; Gallo Cadavid, 2009) que luego se redefinen en el ejercicio de la profesión.

Finalmente, se considera que constituye un importante desafío generar espacios de encuentro y debate entre los docentes de diferentes ámbitos y niveles educativos, con distintas experiencias profesionales a los efectos de compartir inquietudes y generar estrategias que posibiliten resolver problemas propios de cada práctica corporal en especial, y de la educación física en general.

Esta investigación abre camino a una futura revisión de la matriz de contenidos de la formación de grado en Educación Física abrazando las nuevas tendencias y demandas de la sociedad contemporánea.

\title{
EDUCAÇÃO FÍSICA: ENTRE AS NOVAS EXIGÊNCIAS DE PRÁTICA E FORMAÇÃO GRADE
}

\section{Resumo}

O presente artigo apresenta os resultados finais do projeto de pesquisa desenvolvido por uma equipe de pesquisadores do Departamento de Educação Física da Universidade Nacional de Rio Cuarto, durante os anos de 2012 e 2013. O objetivo deste, a pesquisa qualitativa interpretativa foi determinar a relação entre as demandas trabalhistas atuais da prática profissional do ensino de educação física e ensino de graduação. Foram entrevistados em inserções de profundidade a professores que trabalham em diferentes contextos e se dedicam ao ensino de diferentes práticas corporais. A análise destas entrevistas emergiram quatro resultados finais com as suas conclusões, que são detalhados neste artigo.

Palavras-chave: Educação Física; formação de professores; prática profissional; demandas

\section{PHYSICAL EDUCATION: BETWEEN THE NEW DEMANDS OF PRACTICE AND TRAINING GRADE}

\begin{abstract}
The present paper presents the final results of the research project ${ }^{1}$ developed by a research team from the Department of Physical Education, National University of Rio Cuarto during the years 2012 and 2013. The purpose of this qualitative, interpretive research was to determine the relationship between current labor demands of the professional practice of teaching physical education and undergraduate education. Were interviewed in depth inserts occupationally teachers in different work contexts and dedicated to teaching different bodily practices. Analysis of these interviews emerged four final results with their findings, which are detailed in this article.
\end{abstract}

Keywords: Physical education; teacher education; professional practice; demands

\section{Referencias}

BARROW, H., \& BROWN, J. Hombre y Movimiento. España: Doyma, 1992.

BOURDIEU, P. Cosas dichas. Barcelona: Gedisa, 1993. 
BRACHT, V. Educación Física y aprendizaje social. Educación Física/Ciencia del deporte. Que ciencia es esa? Cordoba: Velez Sarsfield, 1996.

BRACHT, V. Educación Física y aprendizaje social. Educación Física/Ciencia del deporte. Que ciencia es esa? Córdoba: Velez Sarsfield, 1996.

BRACHT, V. Educacao Física y Ciencia. Cenas e um casamento (in)feliz. Brasil: Editora Unijuí. Ijuí, 1999.

CACHORRO, G. Ciudad y practicas corporales. La Plata: Universidad Nacional de La Plata, 2013.

CENTURIÓN, S. Educación Física y Universidad. Formación y Práctica. Un Camino entre el Oficio y la profesión. Rio Cuarto: Fundación Universidad Nacional de Río Cuarto, 1997.

CORBIN, S. Y STRAUSS. Bases de la investigación cualitativa. Técnicas y procedimientos para desarrollar la teoría fundamentada. Colombia: Contus Editorial Universidad de Antoquia, 2002.

CRISORIO, R. La teoría de las prácticas. Articulo Inedito, 2007.DAVINI, M. C. La Formación Docente en Cuestión: Política y Pedagogía. Buenos Aires: Paidós, 2005.

DEWEY, J. Democracia y educación. Una introducción a la filosofía de la educación. Madrid: Morata, 1997.

DEWEY, J. Experiencia y educación. La educación tradicional frente a la educación progresiva. Mexico: Losada, 2000.

DI GREGORI, C., \& DURAN, C. Conocimiento y acción: una concepción fundante para el modelo pedagógico y político de John Dewey. Articulo Inedito, 2009.

FENSTERSEIFER, P. Crise da Racionalidade Moderna e a Educação Física. Revista brasilera Ciencias do Esporte, v 2. n ${ }^{\circ}$. p. 35-36, 2000.

GALLO CADAVID, L. Los discursos en la Educación Física contemporánea. Cordoba: Kinesis, 2009.

GAYOL, M. (2013). Educación Física y prácticas corporales curriculares. In: CACHORRO, G.; CAMBLOR, E. (Orgs.), Educación física y ciencias. Abordajes desde la pluralidad. Buenos Aires: Biblos, 2013, p. 235-242. 
GILES, M., MOLINA NETO, V., \& KREUSBURG MOLINA, R. Educación Física y formacion profesional. In: BRACHT, V.; CRISORIO, R. (Orgs.), La Educación Física en Argentina Y Brasil. La Plata: Al Margen, 2005, p. 211-216.

GIRALDES, M. La gimnasia. Buenos Aires: Stadium, 2001.

GLASER, B., \& STRAUSS, A. The discovery of grounded theory strategies for qualitative research. New York: Aldine publishing company, 1967.

GOMEZ CAMPO, V., \& TENTI FANFANI, E. Universidad y Profesores: Crisis y Alternativas. Buenos Aires: Miño y Dávila, 1989.

GUBER, R. La Etnográfia. Métodos, Campo y reflexividad. Bógota: Norma, 2001.

GUTIERREZ PEREZ, J. El proceso de investigación cualitativa desde el enfoque interpretativo de y de la investigacion en acción. In: BUENDÍA, L; GUTIERREZ, J.; GONZALEZ, D.; PELGALAJAR, M. Métodos de análisis de investigación cualitativa. Sevilla: Alfar, 1999, p. 11-59.

KERBRAT ORECCHIONI, C. La enunciación. De la subjetividad al lenguaje. Buenos Aires: $3^{\circ}$ Ed. Edicial, 1997.

LOPES DE PAVIA, F.; RON, O. El campo de la Educacion Fisica. In: CRISORIO, R.; GILES, M. Estudios críticos de la Educación Física La Plata: Al Margen, 2009, p. 5064.

REMEDI, E. El Concepto de Educación Física. Inédito, 1999.

RON, O. El campo de la Educacion Fisica: Constitucion,saber, rasgos. In: BRACHT, V.; CRISORIO R. (Orgs.), LA EDUCACION FISICA EN BRASIL Y ARGENTINA. Identidad, desafios y perspectivas. La Plata: Al Margen, 2005, p. 65-79.

RON, O. ¿Que es la ducación física! Características, lógicas y prácticas. In: CACHORRO, G. ; CAMBLOR, E. (Orgs.), Educación física y ciencias. Abordajes desde la pluralidad. Buenos Aires: Biblos, 2013, p.207-214.

RUIZ, M. Pensando en la situacion actual de la Educ. Fisica en Brasil: formacion e investig. en la Universidad de Rio Grande do Sul. Entrevista a Vicente Molina Neto. Revista digital efdeportes. Año $10 N^{\circ} 76,2004$.

TAYLOR, S.; BOGDAN, R. Introducción a los métodos cualitativos de investigación. Argentina. Buenos Aires: Paidós, 1987. 
TENTI FANFANI, E. El Oficio de Docente. Buenos Aires: Siglo Veintiuno editores, 2005.

VASILACHIS , I. Metódos cualitativos. Los problemas teórico-epistemológico. Buenos Aires: Centro Editores de America Latina, 1992.

VASILACHIS, I. Pobres, pobreza, identidad y representaciones sociales. Barcelona: Gedisa, 2003.

VAZ, A. Educação do corpo, conhecimento, fronteiras. Revista Brasilera de Ciencias do Esporte, Campinas, v. 24, n. 2., 2003, p. 161-172.

Recebido em: 11/08/2014

Revisado em: 29/10/2014

Aprovado em: 02/12/2014

Endereço para correspondência:

ivrivero13@gmail.com

Ivana Verónica Rivero

UNIVERSIDAD NACIONAL DE RÍO CUARTO

RUTA NAC. 36 - KM. 601 / RÍO IV - CÓRDOBA - ARGENTINA 